\title{
ASDEX Upgrade's New Plasma Control Scheme
}

\author{
W. Treutterer*, G. Neu, G. Raupp, D. Zasche, T. Zehetbauer \\ and ASDEX Upgrade Team
}

Max-Planck Institut für Plasmaphysik, EURATOM Association, Boltzmannstrasse 2, D-85748 Garching, Germany

\begin{abstract}
ASDEX Upgrade is a medium sized tokamak experiment investigating highly shaped plasma and advanced scenarios to be extrapolated for ITER. Eleven independent magnetic coils allow for proper shaping and plasma current control. For plasma heating and current drive eight NBI beam lines, two ICRH antenna pairs and four ECRH gyrotrons are available. Five channels for controlling gas valves and a pellet injector serve for fuelling. All actuators are driven by a digital discharge control system. One basic enhancement of the latest generation is a unified framework for all feed-forward and feedback control tasks in a discharge.

The framework consists of two layers. The core layer implements wind-up safe feedback controllers with a collection of overlaid output limitations. Each controller is dynamically switchable in references, controlled variables, control law, and control parameters via a control mode. The coordination layer implements intelligent discharge protection or optimisation algorithms which synchronously can change control modes and dynamically can generate reference waveforms adapted to the discharge's state and goal.

Thus, the framework approach easily allows for further extensions and opens a door for future experimental investigations.
\end{abstract}

Keywords: feedback control, multivariate control, controller coordination, discharge supervision, discharge optimisation

\section{Introduction}

ASDEX Upgrade has been working with digital feedback controllers from the beginning. The first were basic plasma current, position and line averaged electron density control. A supervision process coordinated these control systems by switching between discharge segments dependent on plasma state and time, where each segment contained a collection of pre-programmed waveforms for reference and control mode signals [1].

Over the years a lot of new control systems and variations of existing ones were added to facilitate discharge operation and tune plasma behaviour. Shape control was added for divertor strike point and triangularity control [2], a variety of seven different density controllers evolved, radiated power became feedback controlled and with the aid of a new beta controller discharge scenarios could be stabilised. For quick reaction on unforeseen events a reflex mechanism was introduced [3].

Much expert knowledge from both, theoretical modelling and heuristic experience, flew into the

\footnotetext{
* Corresponding author. Tel ++49/89/3299-1496; fax: ++49/89/3299-2580; email: Treutterer@ipp.mpg.de
} 


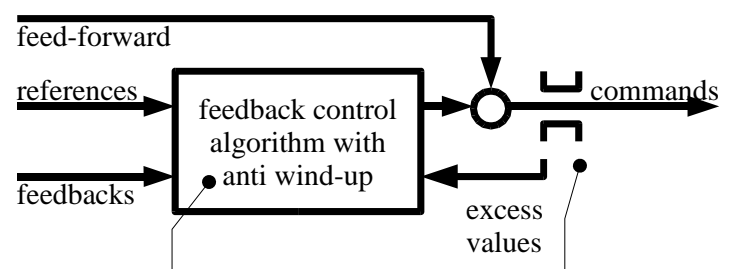

control law : one of

P, PI, PID (scaled),

state space with observer

bang-bang, sliding mode, nonlinear limiters : combination of constant, rate, integral, characteristic curve $\mathrm{f}($ external signals)

\section{Fig. 1. Basic controller}

design of the control algorithms and was cast into tailored software code [4]. These specialised controllers were implemented as standalone solutions. The downside of this encapsulation was, that each implementation not only had its own code, but often also an intrinsic interface, its own sampling rate and overlapping impact with other controllers.

Obviously, sharing common code is difficult under such circumstances and the same applies to maintenance and upgradeability of the whole experiment management. Moreover, the lack of a common interface and the asynchronous operation impede a fluent information exchange, necessary for adapting the feedback topology to the discharge state or for dealing with multivariate control problems.

The latter becomes the more important the more similar the feedback-relevant time constants of a fusion reactor are. A typical example of a multivariate control system is plasma shape control, where the magnetic fields of the actuating coils strongly interfere with the poloidal flux and all actuators have similar response times. Control of current and temperature profile fall into this category, too. Even more, with advanced discharge scenarios additional couplings across the conventional boundaries such as ELM triggering and plasma position modulation must be handled by the control system.

As plasma physics investigations perpetually reveal more of such interdependencies, the upgrade to more multivariate control systems is the appropriate answer. In addition, the quick and synchronous availability of full information on plasma and plant states to all controllers is crucial.

ASDEX Upgrade's new plasma control system has been designed to fulfil these requirements. In [5] it is described, how it is possible to restructure the control loops such, that they share a common interface, re-use highly parametrisable code from a feedback and output limiter algorithm library and outsource measurementand actor-specific treatment. Control law and control parameters are set by a mode switch. Collaboration among the new modules for measurement preprocessing, controlling, monitoring and actuator conditioning is facilitated by the core of the control system - a virtual shared memory - which provides a fast and transparent communication medium for process signals [6].

The improved communication flow allowed also for a reorganisation of the controller coordination. In addition to discharge supervision, reflex handling and waveform interpolation have been centralised and merged such, that intelligent segments can be formed, where adapted waveforms can automatically be generated or modified upon discharge events. With this feature it is not only possible to rescue a marfing discharge or to automate soft landing, it forms the base for the development of real-time discharge optimisation strategies.

As a result the new control scheme comprises two layers. The core layer consists of a number of feedback controllers serving as tools with standardised interface for the superimposed coordination layer. There appropriate control modes and waveforms are generated according to pre-programmed values and knowledge based built-in rules, how to run a discharge.

In the following, we will describe the enhanced features of the new control scheme in more detail.

\section{The core layer}

Core layer feedback controllers have been designed as lean tools focused on the bare feedback task. Measurement pre-processing or specific actuator conditioning have been outsourced. Figure 1 shows the necessary components: feed-forward inputs provide starting points for actuator command outputs and the feedback algorithm calculates output correction values from reference and feedback inputs. In order to allow anti-wind-up strategies in case of output saturation, output value limiters are part of the package, too.

In the course of a discharge several plasma scenarios with differing plasma properties and control goals are traversed. To account for this evolution, controller gains must be adapted, control targets, i.e. reference and feedback inputs, switched and the control algorithm changed. Any such combination is selected 


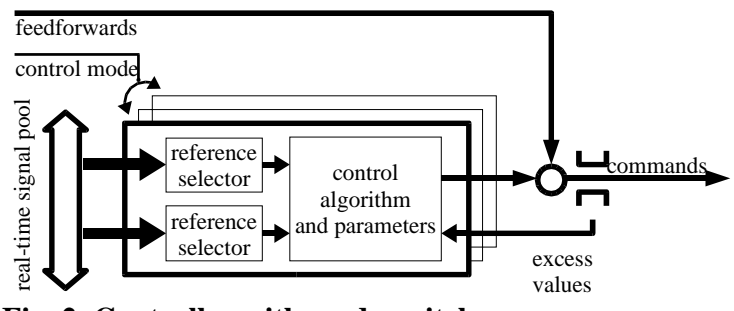

Fig. 2. Controller with mode switch

with a single feedback mode input. If the chosen feedback mode should be not executable for some reason, a fallback mode can be specified.

On the other hand, each controller is invariantly tied to a combination of actuators (fig. 2). Each actuator can be characterised by a feed-forward policy and by a number of limiters, which are subsequently applied to the sum of feed-forward and controller correction values. Dependent on the feed-forward policy, feed-forward inputs can be manipulated to achieve smooth transitions between control modes. Limiters may rely on constant parameters, but also on external signals thus allowing for dynamic limits.

The flexibility of this architecture lies in the variety and extensibility of the control and limiter algorithms. The framework defines the common interface for the coordination layer in form of the control mode descriptor and the associated mode switch signal. The control mode descriptor comprises reference and feedback signals as well as an algorithm identifier. Each control algorithm can define individual parameters like a collection of gains, which are set at configuration time. Thus adding a new algorithm is straightforward.

Presently a standard P, PI and PID control algorithms in scalar and multivariate matrix forms are part of the feedback library. Optionally outputs can be scaled with plasma current. Further extensions with state space controllers with observer and bang-bang controllers are planned. Similarly lots of limiter algorithms are available. They range from constant, rate and integral limiters to multi-dimensional functions of external signals which ASDEX Upgrade uses to avoid exaggerate forces on coil suspensions.

\section{The coordination layer}

ASDEX Upgrade's new control scheme comprises six control systems. Plasma current, position and shape control use magnetic coils as actuators. Fuel control uses gas valves and pellet injection to regulate densities, whereas radiation control doses impurity gas for radiative cooling. Heating control is in charge of energy confinement and - later on - of temperature and current profiles by means of NBI and ICRH (table 1).

\begin{tabular}{|l|l|l|}
\hline $\begin{array}{l}\text { Feedback } \\
\text { controller }\end{array}$ & Controlled variables & Actuators \\
\hline $\begin{array}{l}\text { plasma } \\
\text { current }\end{array}$ & Ip & OH coil (1) \\
\hline $\begin{array}{l}\text { plasma } \\
\text { position }\end{array}$ & (r, z) & fast control coils (2) \\
\hline $\begin{array}{l}\text { plasma } \\
\text { shape }\end{array}$ & $\begin{array}{l}\text { strike points } \\
\text {, gaps, ... }\end{array}$ & $\begin{array}{l}\text { slow poloidal field } \\
\text { coils (8) }\end{array}$ \\
\hline fuel & ne, n0 & $\begin{array}{l}\text { gas channels K1, K2, } \\
\text { K4 }\end{array}$ \\
\hline radiation & $\begin{array}{l}\text { radiated power } \\
\text { divertor temp. }\end{array}$ & gas channels K3, K5 \\
\hline heating & $\begin{array}{l}\text { kpol, } \\
\text { (j, T profiles) }\end{array}$ & $\begin{array}{l}\text { Pheat sum (8 NBI, } \\
\text { 2 ICRH) }\end{array}$ \\
\hline
\end{tabular}

Table 1. Control systems of ASDEX Upgrade

While it is the task of position control to accurately track the reference waveform of e.g. the plasma centre, it is the domain of the coordination layer to select these coordinates as desired control targets and to provide reasonable reference values for them.

Figure 3 shows the coordination layer. It consists of two processes, which are configured by the discharge program. The supervision functionality described in chapter 1 has been ported to the Discharge Monitor process. Its output, the segment number, is used by the second process, the Reference Value Injector to select the appropriate set of waveforms but also an optional waveform pre-processing algorithm which itself is able to generate or modify a set of waveforms. In each control cycle waveforms are interpolated to obtain the actual signal values for control modes, control references and actuator feedforward. Finally these are "injected" into the real-time signal pool from where they are accessible for all control applications.

In normal segments no waveform pre-processing is necessary. The interpolator uses the pre-programmed waveforms from the discharge program. Soft landing after an unforeseen event, however, is a mechanism, where the values must be computed algorithmically with respect to the actual state of the plasma, since the 


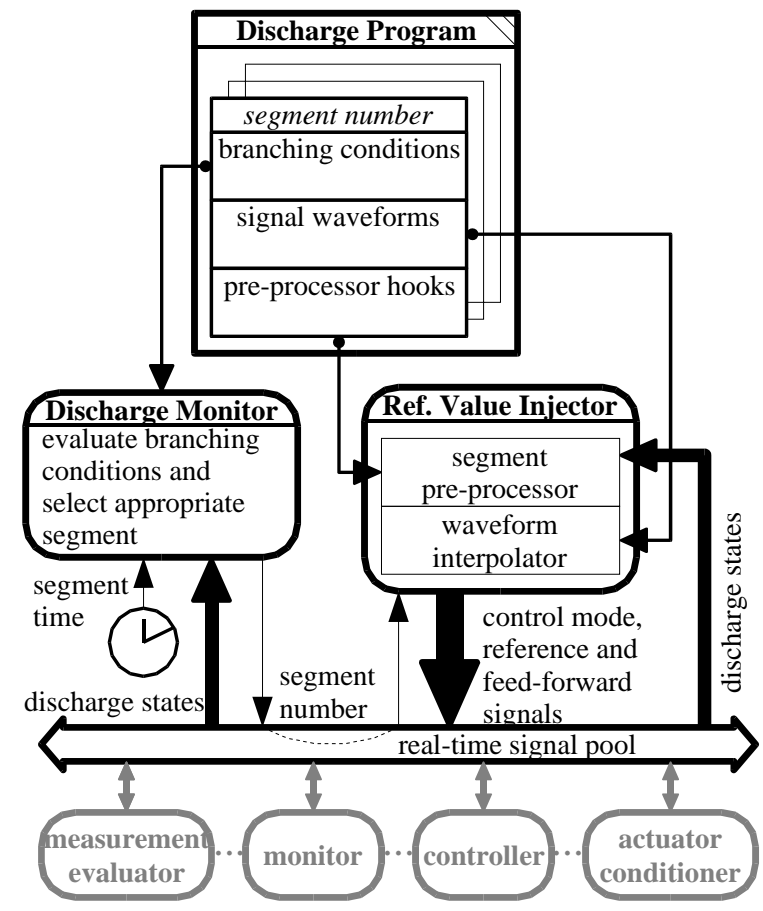

Fig. 3. Coordination laver

initial conditions cannot be predicted in advance. To initiate soft landing the Supervisor selects the corresponding segment. The strength of the coordination layer now is, that it has access to all control systems and can synchronise actions beyond the scope of an isolated controller. The soft landing algorithm, executed by the Injector as segment preprocessor does not only shift plasma to a central position (position control). It transforms it to a circular shape (shape control), while it ramps down the plasma current (current control) and gradually reduces external heating to avoid the density limit (heating control).

Another pre-processor application is marfe protection where normal discharge execution is interrupted in order to apply extra heating until the marfe has disappeared. In this case plasma current, position and shape are frozen, while fuelling is switched off.

Furthermore, the features of the coordination layer have a big potential for future discharge enhancement strategies. Analogously to marfe protection, methods can be implemented to hold or re-establish desired plasma regimes or to iteratively optimise performance indicators.

\section{Conclusion}

The design of ASDEX Upgrade's new plasma control scheme was guided by two major targets. Firstly it should provide a powerful and extensible platform for state-of-the-art discharge control and management methods. Particularly it should account for the increasing knowledge on interdependencies between plasma variables. Secondly a simple and modular structure combined with a virtual shared memory as quick communication medium should ease software usability, maintenance, and future extensions. These requirements resulted in a control framework integrating the feedback control loops in a core layer as executive tools of a superimposed coordination layer.

The new control system has been in regular operation since May 2005. It has successfully been demonstrated, that the features of the previous system could be replicated in the new, restructured scheme. The new capabilities will allow ASDEX Upgrade to boost experimental investigations and to quickly transfer the latest physics knowledge to new control and optimisation algorithms.

\section{References}

[1] G. Raupp et. al. ASDEX-Upgrade Discharge Control and Shot Management. Proc. $17^{\text {th }}$ SOFT, Rome, 1992, p. 1072.

[2] W. Treutterer et. al. Plasma shape control design in ASDEX Upgrade. Proc $19^{\text {th }}$ SOFT, Lisbon, 1996, pp. 933-936.

[3] Raupp et. al. Protection Strategy in the ASDEX Upgrade Control System. Proc. $18^{\text {th }}$ SOFT, Karlsruhe, 1994, pp. 679-682.

[4] V. Mertens, G. Raupp and W. Treutterer. Plasma Control in ASDEX Upgrade. Fusion Science and Technology, Vol. 44, 2003, pp. $593-604$.

[5] W. Treutterer et. al. Plasma Feedback Controller Reorganisation for ASDEX Upgrade's new Discharge Control and Data Acquisition System. Proc. $23^{\text {rd }}$ SOFT, Venice, 2004.

[6] W. Treutterer et. al. The new ASDEX Upgrade RealTime Control and Data Acquisition System. Proc. $22^{\text {rd }}$ SOFT, Helsinki, 2002. 